\title{
Impact of a single-age cohort human papillomavirus vaccination strategy in Catalonia, Spain: Population-based analysis of anogenital warts in men and women
}

\author{
Maria Brotons $^{\mathrm{a}, *}$, Laura Monfil ${ }^{\mathrm{a}}$, Esther Roura ${ }^{\mathrm{a}, \mathrm{b}}$, Talita Duarte-Salles ${ }^{\mathrm{c}}$, Jordi Casabona ${ }^{\mathrm{b}, \mathrm{d}}$, \\ Luis Urbiztondo ${ }^{\mathrm{e}}$, Carmen Cabezas ${ }^{\mathrm{e}}$, F. Xavier Bosch ${ }^{\mathrm{a}, \mathrm{f}}$, Silvia de Sanjosé ${ }^{\mathrm{a}, \mathrm{b}, 1}$, Laia Bruni ${ }^{\mathrm{a}}$ \\ ${ }^{a}$ Unit of Infections and Cancer - Information and Interventions, Cancer Epidemiology Research Programme, Catalan Institute of Oncology (ICO) - IDIBELL, l'Hospitalet de \\ Llobregat, Spain \\ ${ }^{\mathrm{b}}$ Centro de Investigación Biomédica en Red en Epidemiologia y Salud Pública (CIBERESP), Madrid, Spain \\ ${ }^{\mathrm{c}}$ Fundació Institut Universitari per a la recerca a l'Atenció Primària de Salut Jordi Gol i Gurina (IDIAPJGol), Barcelona, Spain \\ ${ }^{\mathrm{d}}$ Centre for Epidemiological Studies of Sexually Transmitted Disease and AIDS in Catalonia (CEEISCAT), Badalona, Spain \\ ${ }^{\mathrm{e}}$ Agència de Salut Pública de Catalunya, Departament de Salut, Generalitat de Catalunya, Barcelona, Spain \\ ${ }^{\mathrm{f}}$ Open University of Catalonia, Barcelona, Spain
}

A R T I C L E I N F O

\section{Keywords:}

HPV

HPV vaccination

Anogenital warts

Incidence

Impact

Time trends

\begin{abstract}
A B S T R A C T
Extensive multiple-age cohort human papillomavirus (HPV) vaccination has proved to be highly effective. We aimed to determine the 8-year population impact of a female single-age cohort HPV vaccination programme on the incidence of anogenital warts (AGW). In 2008, Catalonia initiated a school-based quadrivalent HPV vaccination programme targeting 11-year-old girls, achieving coverage over $80 \%$. Data on diagnoses of AGW and genital herpes were obtained from a population-based database of electronic health records covering $74 \%$ of the population. The annual incidence rates from 2009 to 2016 were calculated, stratified by age and sex using Joinpoint regression to estimate trends and annual percentage changes (APC). Among women aged 16-19 years, the AGW incidence decreased by $61 \%$ from 2012 to 2016 (APC $-19.4 \%$; 95\% CI: -30.0 to -7.3 ). In contrast, the incidence of genital herpes in same-aged women increased throughout the study period (APC $11.1 \%$; 95\% CI: 7.2-15.2). Among men aged 20-22 years, the increasing incidence of AGW shifted to a downward trend in 2013 (APC 2009-2013: 17.0\%; 95\% CI: 8.2-26.5; and APC 2013-2016: -4.5\%; 95\% CI: -14.6 to 6.9). A similar pattern was observed among men aged 23-25 years (APC 2009-2014: 16.0\%; 95\% CI: 12.0-20.2; and APC 2014-2016: $-6.0 \%$; 95\% CI: -18.4 to 8.3). In contrast to AGW, among men aged 20-25 years, the incidence of genital herpes increased over this period. Our study strongly suggests that a single-cohort HPV vaccination strategy with high vaccine uptake not only provides direct benefit in the vaccinated cohorts but also extends protection through a herd effect to unvaccinated men.
\end{abstract}

\section{Introduction}

Anogenital warts (AGW) are the first clinical outcome allowing assessment of the impact of the quadrivalent HPV vaccine, as they occur within few months after HPV infection (Garland et al., 2009). Data from several high-income countries implementing a multiple-age cohort (MAC) quadrivalent HPV vaccination programme have confirmed a fall in AGW diagnoses among targeted girls and women (Ali et al., 2013a; Ali et al., 2013b; Baandrup et al., 2013; Bauer et al., 2012; Cocchio et al., 2017; Dominiak-Felden et al., 2015; Flagg and Torrone, 2018; Leval et al., 2012; Oliphant et al., 2017; Smith et al., 2015; Thöne et al., 2017), with herd effects in men depending on the vaccine coverage (Drolet et al., 2019). However, data on the population-level impact of a single-cohort strategy on the incidence of AGW are limited to a single

${ }^{*}$ Corresponding author at: Unit of Infections and Cancer - Information and Interventions, Cancer Epidemiology Research Programme, Catalan Institute of Oncology (ICO). Av. Granvia 199-203, 08908 L'Hospitalet de Llobregat, Barcelona, Spain.

E-mail addresses: mbrotons@iconcologia.net (M. Brotons), lmonfil@iconcologia.net (L. Monfil), eroura@iconcologia.net (E. Roura), tduarte@idiapjgol.org (T. Duarte-Salles), jcasabona@iconcologia.net (J. Casabona), luis.urbiztondo@gencat.cat (L. Urbiztondo), carmen.cabezas@gencat.cat (C. Cabezas), x.bosch@iconcologia.net (F.X. Bosch), sdesanjose@path.org (S. de Sanjosé), lbruni@iconcologia.net (L. Bruni).

${ }^{1}$ Present address: PATH, Seattle, USA. 
study in Canada, and no herd effects in unvaccinated men have been described to date (Guerra et al., 2016).

In September 2008, Catalonia, a region of 7.5 million inhabitants in northeast Spain, started a school-based, single-age cohort HPV vaccination programme targeting 11-year-old girls and offering the quadrivalent HPV vaccine free of charge, except for the 2010-2011 campaign when the bivalent vaccine was used. More specifically, in 2010-2011, the bivalent vaccine was administered throughout Catalonia except for Barcelona city ( $20 \%$ of girls approximately), where the quadrivalent vaccine was administered due to surplus vaccines from previous years. The use of the quadrivalent vaccine was resumed in 2011-2012 with the exception of a few municipalities that used the surplus bivalent vaccine in that campaign ( $28 \%$ of girls), and continued until 2017-2018 when the nonavalent HPV vaccine was administered. During the first 9 years of the HPV vaccination programme, the overall coverage in Catalonia was over 80\% (Borràs-López et al., 2017; Ministerio de Sanidad, n.d.). In Spain, vaccine uptake outside the programme is uncommon and is estimated to be $<1 \%$ among women up to 45 years of age [Merck Sharp \& Dohme, personal communication].

The quadrivalent HPV vaccine is the most commonly used vaccine in Catalonia and targets high-risk HPV types 16 and 18, accounting for $71 \%$ of cervical cancer cases (de Sanjose et al., 2010), and low-risk HPV types 6 and 11, which cause 90\% of cases of AGW (Garland et al., 2009). The bivalent HPV vaccine protects against HPV 16 and 18, and the nonavalent HPV vaccine provides protection against the same types as the quadrivalent vaccine and against five additional high-risk HPV types, accounting for an additional $20 \%$ of cervical cancer cases (Serrano et al., 2015).

In 2009, the Department of Health of Catalonia and the Catalan Institute of Oncology established a monitoring plan that combined several projects to evaluate the impact of HPV vaccination on disease outcomes. One of these projects evaluates the impact of HPV vaccination on AGW and cervical pre-neoplastic lesions in the public primary care centres of Catalonia. Here we present the first analysis where we evaluate the population impact of the single-age cohort HPV vaccination programme in Catalonia on the incidence of AGW.

\section{Methods}

\subsection{Population}

The study population consisted of all residents of Catalonia aged 10-64 years assigned to a primary care centre of the ICS (Spanish acronym for the Catalan Institute of Health) from 2008 to 2016. The ICS is the main primary health care public provider in Catalonia and offers health care coverage to $>74 \%$ of Catalonian residents. The reference population was the population active in the Information System for Research in Primary Care (SIDIAP database), a population-based database of the anonymised electronic health records of patients assigned to ICS primary care centres that is representative of the population of Catalonia (García-Gil et al., 2011). The study population comprised 4 million individuals each year.

\subsection{Data sources}

The SIDIAP includes validated information on sociodemographic variables, clinical diagnoses coded according to the International Classification of Diseases 10th revision (ICD-10), clinical variables, laboratory test results, and prescriptions and pharmacy invoices coded according to the Anatomical Therapeutic Chemical Classification (ATC) (Bolíbar et al., 2012). Importantly, this comprehensive database includes data from patients seen by general practitioners, by the gynaecologists and midwifes at the network of centres for sexual and reproductive health, and by the network of general practitioners trained as referees for sexually transmitted infections (STI) in primary health care, as well as data from patients seen at the main monographic STI clinic of Catalonia. These publicly-funded services perform all nonhospital based STI diagnoses in the public sector in Catalonia.

\subsection{Case definition}

Between January 2008 and December 2016, all records including the following ICD-10 codes were retrieved: AGW (A63.0), genital Chlamydia infection (A55 and A56), gonorrhoea (A54), and genital herpes (A60). An episode of AGW was defined as incident if it was preceded by a period of at least 12 months with no record of AGW; episodes of genital Chlamydia infection and gonorrhoea were considered incident if they were preceded by a 2-month period with no episodes; and episodes of genital herpes were classified as incident if they were the first episode recorded in the SIDIAP, considering all historical records. Data from the first year of the study period (2008) were used only to exclude prevalent cases. An exploratory analysis showed that the number of gonorrhoea diagnoses among women covered by the HPV vaccination programme (i.e. those younger than 20 years in 2016) was very low, especially in the first 3 years of the study period (5-6 cases diagnosed annually). Additionally, there was a marked rise (3-fold increase) in the incidences of other sexually transmitted chlamydial diseases (A56) between 2014 and 2016, probably reflecting changes in screening strategies, laboratory techniques and reporting tools, which occurred in our setting during this period. Therefore, in this study, we finally used only the incidence of genital herpes as a control disease.

We also obtained data on drugs prescribed for AGW treatment to examine AGW trends, including AGW diagnoses either by identification of an ICD-10 record or a treatment prescription in a sensitivity analysis. We extracted data on prescriptions of podophyllotoxin (ATC code D06BB04), imiquimod (D06BB10), and sinecatechins (D06BB12). Podophyllotoxin and sinecathechins are specific treatments for AGW while imiquimod is also used in actinic keratosis and basal cell carcinoma, conditions that are usually diagnosed in older people. We then restricted the sensitivity analysis to trends in the young population (i.e. persons younger than 30 years). Prescription data were available for 2009 onwards; therefore, to exclude prevalent cases in 2009, we calculated the age- and sex-specific average proportion of prevalent cases identified from prescriptions from 2010 to 2016 and applied these ageand sex- specific proportions to the 2009 data. Since drugs used for treatment of genital herpes are not specific, they were not extracted.

\subsection{Statistical analyses}

We estimated annual incidence rates per 100,000 population with Wald confidence intervals (CI) at $95 \%$ in the population aged 10-64 years. Estimates of incidence rates were stratified by age and sex (5-year age groups from 10 to 64 years, and groups aged 16-19, 20-22, $23-25,26-27$, and $28-29$ years in the analysis of trends in younger cohorts). To assess the effect of switching to the bivalent vaccine in 1 year of the HPV vaccination programme, incidence rate ratios (IRR) were estimated to compare the incidence rate in: i) the last two birth cohorts not covered by the HPV vaccination programme (1995-1996) (reference category); ii) the first two birth cohorts vaccinated with the quadrivalent vaccine (1997-1998); and iii) the only birth cohort vaccinated mainly with the bivalent vaccine (1999).

Analyses were conducted with the Joinpoint Regression Program (version 4.4.0.0) (National Cancer Institute, Bethesda, MD) (National Cancer Institute, n.d.) and the statistical software $\mathrm{R}$ version 3.2.3 (http://www.R-project.org). Joinpoint regression was used to estimate trends in incidence by age and sex, which are reported as the annual percentage change (APC) and its corresponding 95\% CI for each segment. If a single segment is detected, the average annual percentage change (AAPC) equals the APC for that segment. The Joinpoint Regression Program models trends using the Monte Carlo permutation method to select the optimum number of points at which incidence 
trends changed (National Cancer Institute, n.d.). To avoid overfitting due the period length, the maximum number of joinpoints was limited to 1 . A $5 \%$ significance level was used.

This study was approved by the Clinical Research Ethics Committee and the Institutional Review Board of the University Institute for Primary Care Research (IDIAP) Jordi Gol (P15/106).

\section{Results}

\subsection{Overall incidence of $A G W$ and genital herpes}

Between January 1st 2009 and December 31st 2016, a total of 33,933 new AGW cases were identified in individuals aged 10-64 years (15,484 in women and 18,449 in men). The overall incidence rate of AGW among women increased from 72 per 100,000 in 2009 to 107 in 2016, representing an average annual percentage increase of $5.2 \%$ (95\% CI, 2.8-7.6). Among men aged 10-64 years, the incidence of AGW also increased significantly from 69 per 100,000 in 2009 to 135 in 2014 (APC 14.1\%; 95\% CI, 6.8-21.9), and then stabilised (APC 0.6\%; 95\% CI, -21.3 to 28.7) with a resulting rate of 139 per 100,000 in 2016. In 2016, the incidence rate of AGW among women was highest in the group aged 20-24 years, while the incidence among men was highest in the group aged 25-29 years (Fig. 1).

The age- and sex-stratified analysis revealed a statistically significant upward trend in the incidence of AGW from 2009 to 2016 in all age groups except women under 25 years, women aged 60-64 years, and men under 30 years (Supplementary Fig. 1).

During the study period, the overall incidence rate of genital herpes significantly increased in both men and women (APC 9.8\%; 95\% CI, 7.5-12.2, and APC 10.8\%; 95\% CI, 8.8-12.8, respectively) (data not shown).

\subsection{Incidence of $A G W$ and genital herpes among the population under 30 years}

Fig. 2 shows the rates of AGW and genital herpes among young women (Fig. 2A) and men (Fig. 2B) by age group. The incidence of AGW among women aged 16-19 years increased nonsignificantly from 2009 to 2012 (APC 21.1\%; 95\% CI, -0.2 to 46.8) and subsequently significantly declined from 194 per 100,000 in 2012 to 75 in 2016 (61\% reduction; APC $-19.4 \%$; $95 \% \mathrm{CI},-30.0$ to -7.3 ) (Fig. 2A). Supplementary Table 1 shows the potential vaccine received by women aged 16-19 years during the study period. In contrast, the incidence of

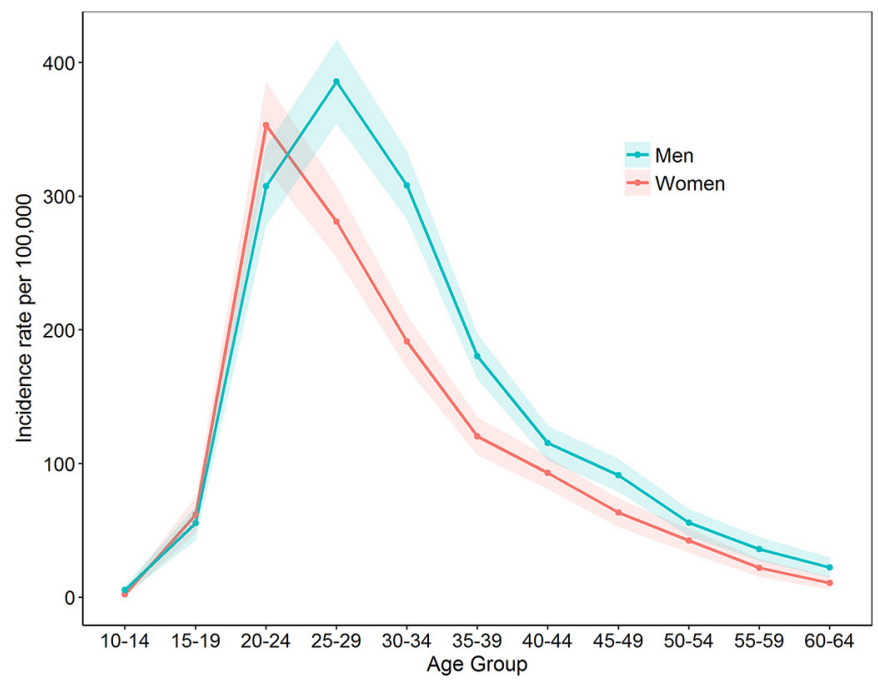

Fig. 1. Age-specific incidence rates of anogenital warts by sex in 2016. Shadowed areas indicate $95 \%$ confidence intervals of the incidence rates. genital herpes among women in the same age group increased significantly from 2009 to 2016 (APC $11.1 \%$; 95\% CI, 7.2-15.2). The incidence rates of both AGW and genital herpes among women aged 20-22 years, 23-25 years, 26-27 years and 28-29 years increased significantly over the study period.

Among men aged 16-19 years, the incidence rates of AGW were quite lower than those among same-aged women and a non-significant increasing trend was observed from 2009 to 2016 (APC 4.2\%; 95\% CI, -3.4 to 12.4) (Fig. 2B). Among men aged 20-22 years, the incidence of AGW significantly increased from 2009 to 2013 (APC 17.0\%; 95\% CI, 8.2-26.5) and then decreased through 2016, although this change was not statistically significant (APC $-4.5 \%$; $95 \% \mathrm{CI},-14.6$ to 6.9 ). A similar pattern was observed among men aged 23-25 years, with a significant increasing trend from 2009 to 2014 (APC 16.0\%; 95\% CI, 12.0-20.2) followed by a non-significant decline through 2016 (APC $-6.0 \%$; $95 \% \mathrm{CI},-18.4$ to 8.3 ). In contrast to these changes in the trends for AGW, rates of genital herpes in both men aged 20-22 years and 23-25 years significantly increased over the study period. The incidence of both AGW and genital herpes significantly increased from 2009 to 2016 among men aged 26-27 years, and the incidence of AGW significantly increased from 2009 to 2013 and then stabilised among men aged 28-29 years, while the incidence of genital herpes increased throughout the study period.

\subsection{Sensitivity analysis}

Table 1 shows the analyses of time trends in the incidence of AGW, including only registered diagnoses and considering both registered diagnoses and/or treatment prescriptions. The addition of confirmed diagnoses through treatment prescriptions increased incident cases by $23 \%$ (during the study period, the incidence rate in individuals aged 16-29 years increased from 235 per 100,000 when registered diagnoses were analysed, to 289 per 100,000 when both registered diagnoses and treatment prescriptions were included) but yielded similar results in the trend analyses. Among women, trend analyses by age group were similar with few exceptions: among women aged 16-19 years the significant downward trend in AGW started a year later (2013) with a somewhat higher average decrease, and among women aged 23-25 years and 28-29 years, the slightly increasing trend was not statistically significant. Among men, the addition of treatment prescriptions resulted in similar trends with the following exceptions: among men aged 16-19 years, there was a non-significant increasing trend from 2009 to 2011, which then stabilised through 2016; among men aged 20-22 years, the decreasing trend from 2013 to 2016 reached statistical significance; among men aged 23-25 years, the increasing trend ended a year sooner, in 2013, and then stabilised; and among men aged 28-29 years, an increasing trend was detected over the study period.

\subsection{Incidence of $A G W$ and genital herpes by birth cohort}

Table 2 shows the age-specific IRR of AGW and genital herpes among women aged 16-19 years in the last two birth cohorts not covered by the HPV vaccination programme (1995-1996), the first two birth cohorts vaccinated with the quadrivalent vaccine (1997-1998), and the only birth cohort vaccinated mainly with the bivalent vaccine (1999). Birth cohorts vaccinated with the quadrivalent vaccine showed a lower incidence of AGW than unvaccinated cohorts at the age of 16, 17,18 and 19 years, whereas no differences were observed in the incidence rates of genital herpes between those cohorts at the same ages. On the other hand, the birth cohort vaccinated mainly with the bivalent vaccine showed a lower incidence of AGW than unvaccinated cohorts; this difference was not statistically significant in individuals aged 16 years but reached statistical significance in those aged 17 years. 

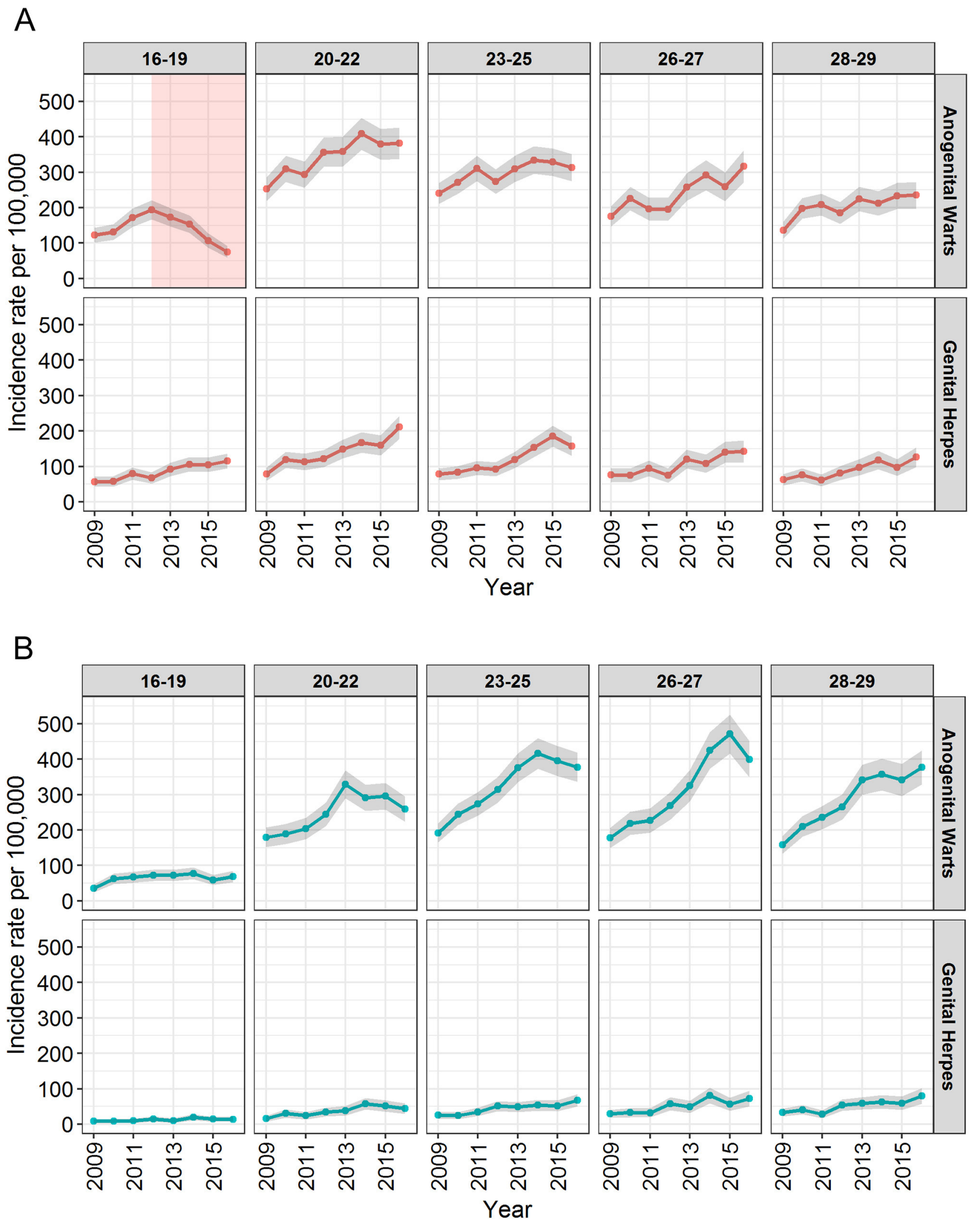

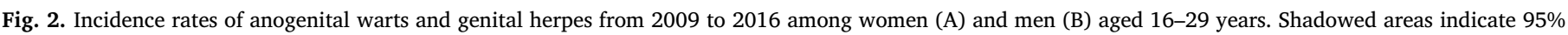

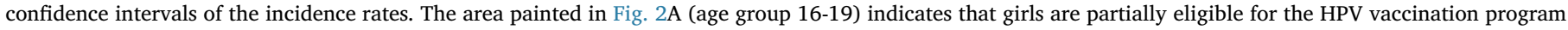

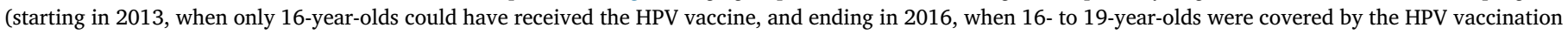
program). 
Table 1

Sensitivity analysis. Comparison of time trends in the incidence of anogenital warts in the young population by age and sex, including only registered diagnoses (ICD10 code A63.0) and both diagnoses and/or treatment prescriptions, 2009-2016.

\begin{tabular}{|c|c|c|c|c|c|c|c|c|}
\hline \multirow[t]{2}{*}{ Age group } & \multicolumn{4}{|c|}{ Trends considering only registered diagnoses } & \multicolumn{4}{|c|}{ Trends considering both registered diagnoses and/or treatment prescriptions } \\
\hline & Trend segment & APC & $95 \%$ CI & p-value & Trend segment & APC & $95 \%$ CI & p-value \\
\hline \multicolumn{9}{|l|}{ Women } \\
\hline \multirow[t]{2}{*}{ 16-19 } & 2009-2012 & 21.1 & $-0.2-46.8$ & 0.051 & $2009-2013$ & 11.7 & $-1.3-26.5$ & 0.066 \\
\hline & $2012-2016$ & -19.4 & $-30--7.3$ & 0.016 & 2013-2016 & -26.1 & $-42.1--5.6$ & 0.029 \\
\hline $20-22$ & $2009-2016$ & 5.8 & $2.6-9.2$ & 0.004 & $2009-2016$ & 4.6 & $1.3-8$ & 0.014 \\
\hline $23-25$ & $2009-2016$ & 3.8 & $0.9-6.7$ & 0.017 & $2009-2016$ & 2.8 & $-0.1-5.8$ & 0.057 \\
\hline $26-27$ & $2009-2016$ & 7.7 & $3.4-12.1$ & 0.004 & $2009-2016$ & 7.1 & $3.2-11.2$ & 0.004 \\
\hline $28-29$ & 2009-2016 & 5.5 & $1.1-10.2$ & 0.022 & $2009-2016$ & 4.6 & $0-9.4$ & 0.051 \\
\hline \multicolumn{9}{|l|}{ Men } \\
\hline \multirow[t]{2}{*}{ 16-19 } & 2009-2016 & 4.2 & $-3.4-12.4$ & 0.232 & $2009-2011$ & 35.4 & $-0.1-83.6$ & 0.051 \\
\hline & & & & & 2011-2016 & -1.5 & $-6.8-4.2$ & 0.457 \\
\hline \multirow[t]{2}{*}{$20-22$} & 2009-2013 & 17 & $8.2-26.5$ & 0.008 & $2009-2013$ & 15.6 & $12.6-18.8$ & $<0.001$ \\
\hline & $2013-2016$ & -4.5 & $-14.6-6.9$ & 0.285 & $2013-2016$ & -4.5 & $-8.1--0.7$ & 0.032 \\
\hline \multirow[t]{2}{*}{$23-25$} & $2009-2014$ & 16 & $12-20.2$ & 0.001 & $2009-2013$ & 17.8 & $8.9-27.4$ & 0.007 \\
\hline & 2014-2016 & -6 & $-18.4-8.3$ & 0.259 & $2013-2016$ & 0.5 & $-9.6-11.8$ & 0.884 \\
\hline $26-27$ & $2009-2016$ & 14.8 & $9.4-20.4$ & $<0.001$ & $2009-2016$ & 14.3 & $10-18.7$ & $<0.001$ \\
\hline \multirow[t]{2}{*}{$28-29$} & $2009-2013$ & 19.4 & $8.9-30.9$ & 0.009 & $2009-2016$ & 12.6 & $8-17.4$ & $<0.001$ \\
\hline & $2013-2016$ & 3.3 & $-9.1-17.3$ & 0.478 & & & & \\
\hline
\end{tabular}

\section{Discussion}

This study demonstrates a substantial reduction in the incidence of AGW among women eligible for the HPV vaccination programme in Catalonia. From 2012 to 2016, AGW incidence rates decreased by $61 \%$ among women aged 16-19 years who were eligible for HPV vaccination, but increased or remained stable among older women, who were all non-eligible for HPV vaccination. Among men, the incidence of AGW showed an upward trend in all groups except those aged 20-22 years and 23-25 years, in which the increasing incidence shifted to a downward trend in 2013 and 2014, respectively. By contrast, the incidence of genital herpes in young men and women showed increasing trends during the study period.

Our findings show a larger decrease in AGW among eligible women than that reported in a study assessing the impact of the single-cohort vaccination programme in Ontario (Canada), which reported a $6.5 \%$ decline in AGW incidence among women aged 18-20 years between pre-vaccination and post-vaccination periods (Guerra et al., 2016). This discrepancy could be explained by differences in the estimation of the reduction, as we calculated the decrease between the last cohorts not eligible for HPV vaccination and the eligible cohorts, rather than that between pre- and post-vaccination periods. The differences may also be due to the lower vaccine uptake in Ontario in the first four years of the programme (51\%-58\%) (Guerra et al., 2016). Our results are similar to those recently reported in a meta-analysis in countries vaccinating MAC with coverage $\geq 50 \%$ after $1-4$ years of vaccine introduction, which found a 64\% reduction among women aged 15-19 years (Drolet et al., 2019). This suggests that the overall effect of single-cohort vaccination programmes could be similar to that of MAC strategies, although the effects in countries adopting a MAC strategy become apparent much sooner. Lastly, our findings are consistent with reductions seen in a study performed in Valencia (Spain) that assessed individual-level vaccination data and reported an effectiveness against AGW of the quadrivalent vaccine (3-dose schedule) of 77\% (Navarro-Illana et al., 2017).

The shift to a downward trend in the incidence of AGW among unvaccinated men aged 20-25 years strongly suggests an early herd effect of the HPV vaccination programme, as the increasing incidence of genital herpes indicate this is unlikely to be explained by changes in sexual behaviour. Although the decreases were not yet statistically significant, the shifts indicate that the increasing trend of AGW among young men has at least been contained. Of note, men aged 16-19 years had a low incidence of AGW with a non-significant time trend suggesting a different pattern of sexual exposure compared with women in the same age category. The age-specific figures for the incidence of AGW by sex (Fig. 1) showed that rates among men were slightly moved to older ages; therefore, it is likely that women eligible for HPV vaccination had older partners, consistent with the results found in our

Table 2

Age-specific incidence rates ratios (IRR) of anogenital warts and genital herpes by birth cohort among women aged 16-19 years.

\begin{tabular}{|c|c|c|c|c|c|c|c|c|c|}
\hline \multirow[t]{3}{*}{ Birth cohort } & \multirow[t]{3}{*}{ Vaccination program } & \multicolumn{8}{|c|}{ Age (years) } \\
\hline & & \multicolumn{2}{|r|}{16} & \multicolumn{2}{|r|}{17} & \multicolumn{2}{|r|}{18} & \multicolumn{2}{|r|}{19} \\
\hline & & $\mathrm{N}$ cases & IRR (95\% CI) & $\mathrm{N}$ cases & IRR $(95 \% \mathrm{CI})$ & $\mathrm{N}$ cases & IRR (95\% CI) & $\mathrm{N}$ cases & IRR (95\% CI) \\
\hline \multicolumn{10}{|c|}{ Anogenital warts } \\
\hline $1995-1996^{a}$ & No vaccination & 31 & 1.00 (Ref) & 67 & 1.00 (Ref) & 103 & 1.00 (Ref) & 157 & 1.00 (Ref) \\
\hline $1997-1998^{b}$ & Quadrivalent & 11 & $0.36(0.17,0.69)$ & 18 & $0.27(0.16,0.45)$ & 34 & $0.33(0.22,0.48)$ & 27 & $0.34(0.22,0.50)$ \\
\hline $1999^{c}$ & Mainly bivalent & 9 & $0.57(0.25,1.15)$ & 20 & $0.58(0.34,0.94)$ & - & - & - & - \\
\hline \multicolumn{10}{|l|}{ Genital herpes } \\
\hline $1995-1996^{\mathrm{a}}$ & No vaccination & 13 & 1.00 (Ref) & 33 & 1.00 (Ref) & 73 & 1.00 (Ref) & 72 & 1.00 (Ref) \\
\hline $1997-1998^{b}$ & Quadrivalent & 22 & $1.68(0.86,3.45)$ & 40 & $1.21(0.76,1.93)$ & 63 & $0.86(0.61,1.20)$ & 41 & $1.11(0.75,1.61)$ \\
\hline $1999^{c}$ & Mainly bivalent & 13 & $1.94(0.88,4.25)$ & 26 & $1.52(0.90,2.55)$ & - & - & - & - \\
\hline
\end{tabular}

a Not covered by the HPV vaccination program.

b Quadrivalent HPV vaccine eligible (estimated 3-dose coverage 80\%).

c $78 \%$ of women bivalent HPV vaccine eligible; $22 \%$ of women quadrivalent HPV vaccine eligible (estimated 3-dose coverage $80 \%$ ). 
study. Herd effects of HPV vaccination programmes in unvaccinated men have been widely reported in high-income countries that vaccinated MAC (Ali et al., 2013a; Ali et al., 2013b; Bauer et al., 2012; Dominiak-Felden et al., 2015; Flagg and Torrone, 2018; Smith et al., 2015; Thöne et al., 2017; Bollerup et al., 2016) but, to our knowledge, this is the first description of herd effects in unvaccinated men following a single-cohort strategy. Drolet et al. (2019) stated in their metaanalysis that the magnitude of the decreases observed in unvaccinated men depends on the overall proportion of people vaccinated in the country, with no herd effects observed in men in countries with a low proportion of vaccinated individuals (countries with a single-cohort strategy or those with a MAC strategy and coverage under 50\%). Furthermore, a modelling study including data from Australia showed the importance of catch-up cohorts in the achievement of herd effects (Drolet et al., 2017). While we were able to observe the extended benefit of a single-cohort HPV vaccination strategy in unvaccinated men, vaccinating additional cohorts could have had an additional, faster impact in the population.

We also aimed to assess the effect of switching to the bivalent vaccine in one year of the vaccination programme on the incidence of AGW. We observed a significantly lower rate of AGW among 17-yearolds, which could have several explanations. First, the cohort eligible for the bivalent vaccine was not fully vaccinated with this vaccine, as $22 \%$ of women received the quadrivalent vaccine. Therefore, the birth cohort was partially protected against AGW. Second, in this study, we found herd effects in unvaccinated young men, raising the possibility that females in the cohort who received primarily the bivalent vaccine also received some herd protection from the quadrivalent vaccine given to other cohorts. Lastly, the bivalent vaccine may have a protective effect against AGW itself. Two ecological studies conducted in England have suggested that the bivalent vaccine protects against AGW, showing decreases in the incidence of AGW among young girls and young heterosexual men that were associated with HPV vaccine coverage (Canvin et al., 2017; Howell-Jones et al., 2013). By contrast, studies assessing individual-level data on HPV vaccination in Spain and the Czech Republic did not confirm a protective effect against AGW (Navarro-Illana et al., 2017; Petráš and Adámková, 2015).

\subsection{Study limitations and strengths}

Our data cover only the public sector, and therefore the actual incidence rates may be underestimated. In Catalonia, it is estimated that $29 \%$ of women and $30 \%$ of men aged 15-44 years have dual health insurance coverage (i.e. both public and private health insurance) (Generalitat de Catalunya, Departament de Salut, 2019). We performed a sensitivity analysis including AGW diagnoses identified from treatment prescriptions, as it is not uncommon for people visiting private physicians to seek drug treatments covered by the publicly-funded health system. The sensitivity analyses showed that $23 \%$ of incident AGW cases diagnosed in young people had not been registered, but yielded similar trends in vaccinated cohorts and in men aged 20-25 years. Therefore, considering the results of the sensitivity analysis and that the underestimation applies to the entire study period, we believe it is unlikely that our results were affected by this potential limitation. Furthermore, the herd effects observed in men were probably limited by the lack of data on sexual orientation in the SIDIAP database. If we had been able to perform the analysis in men by sexual orientation, we would have expected a greater decrease in heterosexual men, with no decreases in homosexual men, as described by others (Ali et al., 2013a; Chow et al., 2015).

Our study also has several strengths. First, we used a representative database covering $74 \%$ of the general Catalonian population (GarcíaGil et al., 2011). In addition, the database included medical records not only from general practitioners, but also from all the sexual and reproductive health services and STI clinics. Moreover, using genital herpes as a well-documented control STI allowed us to differentiate vaccination impact from potential changes in exposure over time. Finally, our analyses relied on registered clinical diagnoses of AGW, not AGW treatments, which is important as the temporal trend analysis was not influenced by changes in clinical practice during the study period.

\section{Conclusion}

We found that AGW substantially decreased among vaccinated cohorts, confirming the early impact of a single-age cohort HPV vaccination strategy. The shift to a downward trend in AGW in young men strongly suggests an early herd effect of single-cohort HPV vaccination. Although a MAC vaccination strategy is recommended to increase the benefits of the HPV vaccination programme (World Health Organization, 2017), the introduction of MAC may be hindered by several factors, including affordability or problems with HPV vaccine supply (UNICEF, 2018; WHO, 2019). Our results could help to envisage the impact of a single-cohort vaccination programme, where a MAC is currently not a feasible option.

Supplementary data to this article can be found online at https:// doi.org/10.1016/j.ypmed.2020.106166.

\section{CRediT authorship contribution statement}

Maria Brotons: Conceptualization, Methodology, Writing - original draft. Laura Monfil: Software, Formal analysis, Visualization. Esther Roura: Software, Formal analysis, Validation. Talita DuarteSalles: Writing - review \& editing. Jordi Casabona: Writing - review \& editing. Luis Urbiztondo: Funding acquisition, Writing - review \& editing. Carmen Cabezas: Funding acquisition, Writing - review \& editing. F. Xavier Bosch: Writing - review \& editing. Silvia de Sanjosé: Writing - review \& editing. Laia Bruni: Conceptualization, Supervision, Writing - review \& editing.

\section{Declaration of competing interest}

F. Xavier Bosch has received scientific advisory board fees, speaker's fees, or travel grants from GlaxoSmithKline, Merck and Co., Inc. and IMS Health and unrestricted institutional research grants from GlaxoSmithKline and Merck and Co., Inc. The Cancer Epidemiology Research Programme (with which Maria Brotons, Laura Monfil, Esther Roura, F. Xavier Bosch, Silvia de Sanjosé and Laia Bruni are affiliated) has received sponsorship for grants from Merck and Co., Inc. and GSK. The rest of the authors declare no conflicts of interest.

\section{Acknowledgements}

To Xavi Castellsagué, who initiated this project and passed away in 2016. We acknowledge the SIDIAP, with special thanks to Maria Aragon for technical support. The results of the "Monitorización y evaluación del impacto de la introducción de nuevas estrategias preventivas del cancer de cuello de útero en Catalunya" (0599S/7613/2010) project are partially funded by the Department of Health of the Generalitat de Catalunya. This study has been partially funded by Instituto de Salud Carlos III through grant CM15/00061 Río Hortega (co-funded by European Social Fund. ESF, investing in your future) and through the CIBERESP CB06/02/0073 project (co-funded by European Regional Development Fund. ERDF, a way to build Europe). With the support of the Secretariat for Universities and Research of the Department of Business and Knowledge of the Government of Catalonia, grants to support the activities of research groups (SGR 2017-2019) number 2017SGR1718 and 2017SGR1085. We also thank CERCA Programme/ Generalitat de Catalunya for institutional support. The funding sources had no role in the study design, data collection, analysis or interpretation of the results, writing of the manuscript, or the decision to submit the manuscript for publication. 


\section{References}

Ali, H., Donovan, B., Wand, H., Read, T.R.H., Regan, D.G., Grulich, A.E., et al., 2013a Genital warts in young Australians five years into national human papillomavirus vaccination programme: national surveillance data. BMJ 346, f2032. https://doi.org/ 10.1136/bmj.f2032.

Ali, H., Guy, R.J., Wand, H., Read, T.R., Regan, D.G., Grulich, A.E., et al., 2013b. Decline in in-patient treatments of genital warts among young Australians following the national HPV vaccination program. BMC Infect. Dis. 13, 140. https://doi.org/10. 1186/1471-2334-13-140.

Baandrup, L., Blomberg, M., Dehlendorff, C., Sand, C., Andersen, K.K., Kjaer, S.K., 2013. Significant decrease in the incidence of genital warts in young Danish women after implementation of a national human papillomavirus vaccination program. Sex. Transm. Dis. 40, 130-135. https://doi.org/10.1097/OLQ.0b013e31827bd66b.

Bauer, H.M., Wright, G., Chow, J., 2012. Evidence of human papillomavirus vaccineeffectiveness in reducing genital warts: an analysis of California public family planning administrative claims data, 2007-2010. Am. J. Public Health 102, 833-835. https://doi.org/10.2105/AJPH.2011.300465.

Bolíbar, B., Fina Avilés, F., Morros, R., Garcia-Gil, M. del M., Hermosilla, E., Ramos, R., et al., 2012. SIDIAP database: electronic clinical records in primary care as a source of information for epidemiologic research. Med. Clin. (Barc.) 138, 617-621. https://doi. org/10.1016/j.medcli.2012.01.020.

Bollerup, S., Baldur-Felskov, B., Blomberg, M., Baandrup, L., Dehlendorff, C., Kjaer, S.K., 2016. Significant reduction in the incidence of genital warts in young men 5 years into the Danish Human Papillomavirus Vaccination Program for girls and women. Sex. Transm. Dis. 43, 238-242. https://doi.org/10.1097/OLQ.0000000000000418.

Borràs-López, E., Urbiztondo-Perdices, L., Martínez-Marcos, M., Ibáñez, D., Cabezas-Peña, C., 2017. La vacunació contra el virus del papillloma humà a Catalunya: cobertures i notificacions de sospita de reaccions adverses a vacunes. Butll Epidemiol. Catalunya 36, 137-143.

Canvin, M., Sinka, K., Hughes, G., Mesher, D., 2017. Decline in genital warts diagnoses among young women and young men since the introduction of the bivalent HPV (16/ 18) vaccination programme in England: an ecological analysis. Sex. Transm. Infect. 93, 125-128. https://doi.org/10.1136/sextrans-2016-052626.

Chow, E.P.F., Read, T.R.H., Wigan, R., Donovan, B., Chen, M.Y., Bradshaw, C.S., et al., 2015. Ongoing decline in genital warts among young heterosexuals 7 years after the Australian human papillomavirus (HPV) vaccination programme. Sex. Transm. Infect. 91, 214-219. https://doi.org/10.1136/sextrans-2014-051813.

Cocchio, S., Baldovin, T., Bertoncello, C., Buja, A., Furlan, P., Saia, M., et al., 2017. Decline in hospitalization for genital warts in the Veneto region after an HPV vaccination program: an observational study. BMC Infect. Dis. 17, 249. https://doi.org/ 10.1186/s12879-017-2361-5.

de Sanjose, S., Quint, W.G., Alemany, L., Geraets, D.T., Klaustermeier, J.E., Lloveras, B., et al., 2010. Human papillomavirus genotype attribution in invasive cervical cancer: a retrospective cross-sectional worldwide study. Lancet Oncol. 11, 1048-1056. https://doi.org/10.1016/S1470-2045(10)70230-8.

Dominiak-Felden, G., Gobbo, C., Simondon, F., 2015. Evaluating the early benefit of quadrivalent HPV vaccine on genital warts in Belgium: a cohort study. PLoS One 10, e0132404. https://doi.org/10.1371/journal.pone.0132404.

Drolet, M., Laprise, J.-F., Brotherton, J.M.L., Donovan, B., Fairley, C.K., Ali, H., et al., 2017. The impact of human papillomavirus catch-up vaccination in Australia: implications for introduction of multiple age cohort vaccination and post-vaccination data interpretation. J. Infect. Dis. 216, 1205-1209. https://doi.org/10.1093/infdis/ jix476.

Drolet, M., Bénard, É., Pérez, N., Brisson, M., Ali, H., Boily, M.-C., et al., 2019. Populationlevel impact and herd effects following the introduction of human papillomavirus vaccination programmes: updated systematic review and meta-analysis. Lancet 394 , 497-509. https://doi.org/10.1016/S0140-6736(19)30298-3.

Flagg, E.W., Torrone, E.A., 2018. Declines in anogenital warts among age groups most likely to be impacted by human papillomavirus vaccination, United States, 2006-
2014. Am. J. Public Health 108, 112-119. https://doi.org/10.2105/AJPH.2017. 304119.

García-Gil, M.D.M., Hermosilla, E., Prieto-Alhambra, D., Fina, F., Rosell, M., Ramos, R., et al., 2011. Construction and validation of a scoring system for the selection of highquality data in a Spanish population primary care database (SIDIAP). Inform. Prim. Care 19, 135-145. https://doi.org/10.14236/jhi.v19i3.806.

Garland, S.M., Steben, M., Sings, H.L., James, M., Lu, S., Railkar, R., et al., 2009. Natural history of genital warts: analysis of the placebo arm of 2 randomized phase III trials of a quadrivalent human papillomavirus (types $6,11,16$, and 18 ) vaccine. J. Infect. Dis. 199, 805-814. https://doi.org/10.1086/597071.

Generalitat de Catalunya, Departament de Salut, 2019. Enquesta de Salut de Catalunya 2018. Principals indicadors ESCA2018.

Guerra, F.M., Rosella, L.C., Dunn, S., Wilson, S.E., Chen, C., Deeks, S.L., 2016. Early impact of Ontario's human papillomavirus (HPV) vaccination program on anogenital warts (AGWs): a population-based assessment. Vaccine 34, 4678-4683. https://doi. org $/ 10.1016 /$ j.vaccine. 2016.08.020.

Howell-Jones, R., Soldan, K., Wetten, S., Mesher, D., Williams, T., Gill, O.N., et al., 2013. Declining genital warts in young women in England associated with HPV 16/18 vaccination: an ecological study. J. Infect. Dis. 208, 1397-1403. https://doi.org/10. 1093/infdis/jit361.

Leval, A., Herweijer, E., Arnheim-Dahlström, L., Walum, H., Frans, E., Sparén, P., et al., 2012. Incidence of genital warts in Sweden before and after quadrivalent human papillomavirus vaccine availability. J. Infect. Dis. 206, 860-866. https://doi.org/10. 1093/infdis/jis405.

Ministerio de Sanidad, Consumo y Bienestar Social. Histórico de coberturas de vacunación n.d. https://www.mscbs.gob.es/profesionales/saludPublica/ prevPromocion/vacunaciones/HistoricoCoberturas.htm (accessed May 2, 2020).

National Cancer Institute. Joinpoint. n.d.

Navarro-Illana, E., López-Lacort, M., Navarro-Illana, P., Vilata, J.J., Diez-Domingo, J., 2017. Effectiveness of HPV vaccines against genital warts in women from Valencia, Spain. Vaccine 35, 3342-3346. https://doi.org/10.1016/j.vaccine.2017.04.080.

Oliphant, J., Stewart, J., Saxton, P., Lo, M., Perkins, N., Ward, D., 2017. Trends in genital warts diagnoses in New Zealand five years following the quadrivalent human papillomavirus vaccine introduction. N. Z. Med. J. 130, 9-16.

Petráš, M., Adámková, V., 2015. Impact of quadrivalent human papillomavirus vaccine in women at increased risk of genital warts burden: population-based cross-sectional survey of Czech women aged 16 to 40 years. Vaccine 33, 6264-6267. https://doi. org/10.1016/j.vaccine.2015.09.071.

Serrano, B., de Sanjosé, S., Tous, S., Quiros, B., Muñoz, N., Bosch, X., et al., 2015. Human papillomavirus genotype attribution for HPVs $6,11,16,18,31,33,45,52$ and 58 in female anogenital lesions. Eur. J. Cancer 51, 1732-1741. https://doi.org/10.1016/j. ejca.2015.06.001.

Smith, M.A., Liu, B., McIntyre, P., Menzies, R., Dey, A., Canfell, K., 2015. Fall in genital warts diagnoses in the general and indigenous Australian population following implementation of a National Human Papillomavirus Vaccination Program: analysis of routinely collected national hospital data. J. Infect. Dis. 211, 91-99. https://doi.org/ 10.1093/infdis/jiu370.

Thöne, K., Horn, J., Mikolajczyk, R., 2017. Evaluation of vaccination herd immunity effects for anogenital warts in a low coverage setting with human papillomavirus vaccine-an interrupted time series analysis from 2005 to 2010 using health insurance data. BMC Infect. Dis. 17, 564. https://doi.org/10.1186/s12879-017-2663-7.

UNICEF, 2018. Supply Division. Human Papillomavirus Vaccine Supply and Demand Update. Copenhagen, UNICEF.

WHO, 2019. Meeting of the Strategic Advisory Group of Experts on Immunization, October 2019: conclusions and recommendations. Wkly Epidemiol. Rec. 94, 550-551.

World Health Organization, 2017. Human papillomavirus vaccines: WHO position paper, May 2017-recommendations. Vaccine 35, 5753-5755. https://doi.org/10.1016/j. vaccine.2017.05.069. 